OPEN ACCESS

Edited by:

Walter Adriani

Istituto Superiore di Sanità (ISS), Italy

Reviewed by:

Eleni Konsolaki,

American College of Greece, Greece Jana Ruda-Kucerova,

Masaryk University, Czechia

*Correspondence:

Silvia Mandillo

silvia.mandillo@cnr.it

†These authors have contributed equally to this work

Received: 17 August 2018 Accepted: 24 October 2018 Published: 13 November 2018

Citation:

Garbugino L, Golini E, Giuliani A and Mandillo S (2018) Prolonged Voluntary Running Negatively Affects Survival and Disease Prognosis of Male SOD1G93A Low-Copy Transgenic Mice. Front. Behav. Neurosci. 12:275 doi: 10.3389/fnbeh.2018.00275

\section{Prolonged Voluntary Running Negatively Affects Survival and Disease Prognosis of Male SOD1G93A Low-Copy Transgenic Mice}

\author{
Luciana Garbugino $^{1 \dagger}$, Elisabetta Golini ${ }^{1+}$, Alessandro Giuliani ${ }^{2}$ and Silvia Mandillo ${ }^{1 *}$ \\ ${ }^{1}$ Istituto di Biologia Cellulare e Neurobiologia, Consiglio Nazionale delle Ricerche, Rome, Italy, ${ }^{2}$ Environment and Health \\ Department, Istituto Superiore di Sanità, Rome, Italy
}

Amyotrophic Lateral Sclerosis (ALS) is a disease in which physical activity plays a controversial role. Epidemiological studies indicate an association between intense exercise and risk of developing ALS. To study the impact of physical activity on ALS, mouse models rely mostly on forced exercise. In this study we hypothesized that voluntary wheel running could represent a better model of the influence of exercise in the pathogenesis of ALS. We used an automated home-cage running-wheel system that enables individual monitoring of performance. To verify the effect of voluntary running on disease progression, prognosis and survival as well as motor functions, we challenged SOD1G93A low-copy male and female mice on one (1 RW, at age 24 weeks) or multiple (3 RW) running sessions at age 13, 18, and 24 weeks. In parallel we measured performance on Rotarod and Grip strength tests at different ages. Several parameters were analyzed through Principal Component Analysis in order to detect what indices correlate and may be useful for deeper understanding of the relation between exercise and disease development. We found mutant male mice more negatively affected than females by prolonged and repeated exercise. SOD1G93A low-copy male mice showed shorter survival, increased body weight loss and poorer disease prognosis when exposed to multiple running sessions. These findings could encourage the investigation of the pathogenetic mechanisms underlying the supposedly increased risk to develop ALS in humans engaged in specific and intense exercise activities.

Keywords: ALS, exercise, running wheels, sex differences, rotarod, Principal Component Analysis

\section{INTRODUCTION}

Amyotrophic lateral sclerosis (ALS) is a fatal, multi-systemic disease in which motor neurons degenerate relentlessly affecting the neuromuscular system with a rapid progression that ends in paralysis and death for respiratory failure. Beside known genetic mutations, which are discovered at an increasing rate in both familial and sporadic ALS forms (Ajroud-Driss and Siddique, 2015; Al-Chalabi et al., 2017), a number of environmental risk factors have been associated to the disease: exposure to toxins, pesticides, heavy metals, diet, smoking, anti-inflammatory drugs, doping, and strenuous exercise (Al-Chalabi and Hardiman, 2013; Oskarsson et al., 2015; Bozzoni et al., 2016). However, it is still controversial whether exercise has beneficial or detrimental effects on disease onset and progression. Understanding the impact of physical activity in ALS is thus crucial. It seems 
that in humans, excessive exercise would affect a predisposed genetic profile; recent studies indicate a predisposition toward an "athletic" phenotype that increases susceptibility to ALS (Huisman et al., 2013). While several animal studies rely on forced exercise, we propose that voluntary physical activity (i.e., wheel running) could represent a better tool to model the influence of environmental factors in the pathogenesis of ALS. When considered as a model of human leisure physical activity, voluntary running in mice also provides a reliable representation of individual variability. We hypothesize that prolonged voluntary running could be detrimental in predisposed individuals (i.e., SOD1 mutant mice) and worsen disease prognosis.

We investigated the effects of wheel running in a genetic mouse model of ALS, B6.Cg-Tg(SOD1*G93A) ${ }^{\mathrm{dl}} 1 \mathrm{Gur} / \mathrm{J}$ (SOD1G93A low-copy) expressing about 8-10 copies of the transgene, $30 \%$ fewer copies than the standard SOD1G93A model (Gurney et al., 1994). These mice show delayed onset and slower disease progression and develop paralysis between 28 and 34 weeks of age. This model, used in longitudinal studies, gives the opportunity to examine the very early stages of pathology and allows a longer therapeutic window to test new interventions (Alexander et al., 2004; Acevedo-Arozena et al., 2011). This strain has been already analyzed in our lab with an automated running wheel system and has shown motor deficits as early as 12 weeks of age (Mandillo et al., 2014).

The aim of this study was to verify the effect of voluntary wheel running on the disease onset, progression, motor function (muscular strength, motor coordination and running) and survival of male and female SOD1G93A low-copy mice by challenging them with one or multiple 3-weeks running sessions. We consider very important to focus on the analysis of both male and female individuals as in this and other models of neurodegeneration, the incidence of the disease is often different in the two sexes and very few animal studies tackle this issue (Clayton and Collins, 2014).

Several parameters including motor behavior were analyzed through Principal Component Analysis in order to detect what indices of the different tests may be correlated and useful to understand the impact of exercise on the development of the disease.

We expected wheel running activity, behavioral performance and survival to be differentially altered in male and female SOD1G93A low-copy mice depending on exposure to previous running sessions. In particular, mutant male mice exposed to 3 running wheel sessions showed increased body weight loss, shorter survival and poorer disease prognosis.

\section{MATERIALS AND METHODS}

\section{Animals and Husbandry}

Adult male mice of B6.Cg-Tg(SOD1*G93A) $)^{\mathrm{dl}} 1 \mathrm{Gur} / \mathrm{J}$ (SOD1G93A low-copy) on a C57BL/6J background were initially obtained from MRC (Harwell, UK) and subsequently bred in-house at the CNR-EMMA facility (Monterotondo, Italy) by crossing hemizygous transgenic males with C57BL/6J females. Male and female mice obtained from three consecutive generations were employed in the study. Wild type littermates
(WT) were used as controls. Mice transgenic for the human SOD1G93A mutation represent a widely used model of Amyotrophic Lateral Sclerosis (ALS). Compared to the most used B6.Cg-Tg(SOD1*G93A)1Gur/J transgenics (Gurney et al., 1994), which carry $\sim 25$ copies of the human transgene, SOD1G93A low-copy mice carry $\sim 8-10$ copies, and show a later onset of pathology and prolonged survival (Alexander et al., 2004; Acevedo-Arozena et al., 2011).

Sex and age matched littermates were group-housed, 35 per cage, in standard cages (Thoren, Hazleton, PA, USA) enriched with a transparent red polycarbonate igloo house (Datesand, Manchester, UK) and with wood shavings contained in single cellulose bags (Scobis Uno bags, Mucedola, Settimo Milanese, Italy). Food (2918 Teklad diet, Mucedola, Settimo Milanese, Italy) and water were available ad libitum. Room temperature was $21 \pm 2^{\circ} \mathrm{C}$, relative humidity was $50-60 \%$, and mice were kept in a $12 \mathrm{~h}$ light/dark cycle with lights on at 8 am. Animals were subjected to experimental protocols approved by the Veterinary Dept. of the Italian Ministry of Health, and experiments were conducted according to the ethical and safety rules and guidelines for the use of animals in biomedical research provided by the relevant Italian laws and European Union's directives (Italian Legislative Decree 26/2014 and 2010/63/EU). All adequate measures were taken to minimize animal pain or discomfort.

\section{Experimental Design and Groups}

Mice were divided in three experimental groups for different exposure to exercise: (i) no exposure (no RW), (ii) 1 running session in which mice were exposed to one running wheel session at the age of 24 weeks (1 RW) and (iii) 3 running sessions in which mice were exposed to three running wheel sessions at the age of 13, 18, and 24 weeks ( 3 RW). Each running session lasted 3 weeks. Mice of the "no RW" group were left undisturbed in their home cages and used for comparison with the exercise exposed mice. Mice exposed to running wheels were also tested on rotarod and grip strength tests in between ( $3 \mathrm{RW}$ group) or before and after (1 RW group) running wheel sessions (Figure 1A). The number of mice tested in each experimental group are reported in Figure 1A.

\section{Wheel Running Activity}

During the running wheel sessions, mice were single-housed and a running wheel (TSE Systems, Bad Homburg, Germany) was placed in each home cage (Type II long, Ebeco, Germany) in a dedicated room with the same light/dark cycle and environmental conditions as in the housing room. Data of 3 consecutive weeks of wheel running activity were automatically collected and processed with PhenoMaster software (TSE Systems). In week 1 and week 2, mice were exposed to a standard wheel, while during week 3 a "complex" wheel was introduced. This wheel has a fewer number of rods which are irregularly spaced, though with a predictable pattern (Liebetanz and Merkler, 2006; Mandillo et al., 2014). Food and water were available ad libitum, and nestpaper was provided. Cages were changed every week. Wheel running activity for the $12 \mathrm{~h}$ dark phase was included in this study. Parameters measured were weekly values of: (1) total distance (m); (2) average run duration 


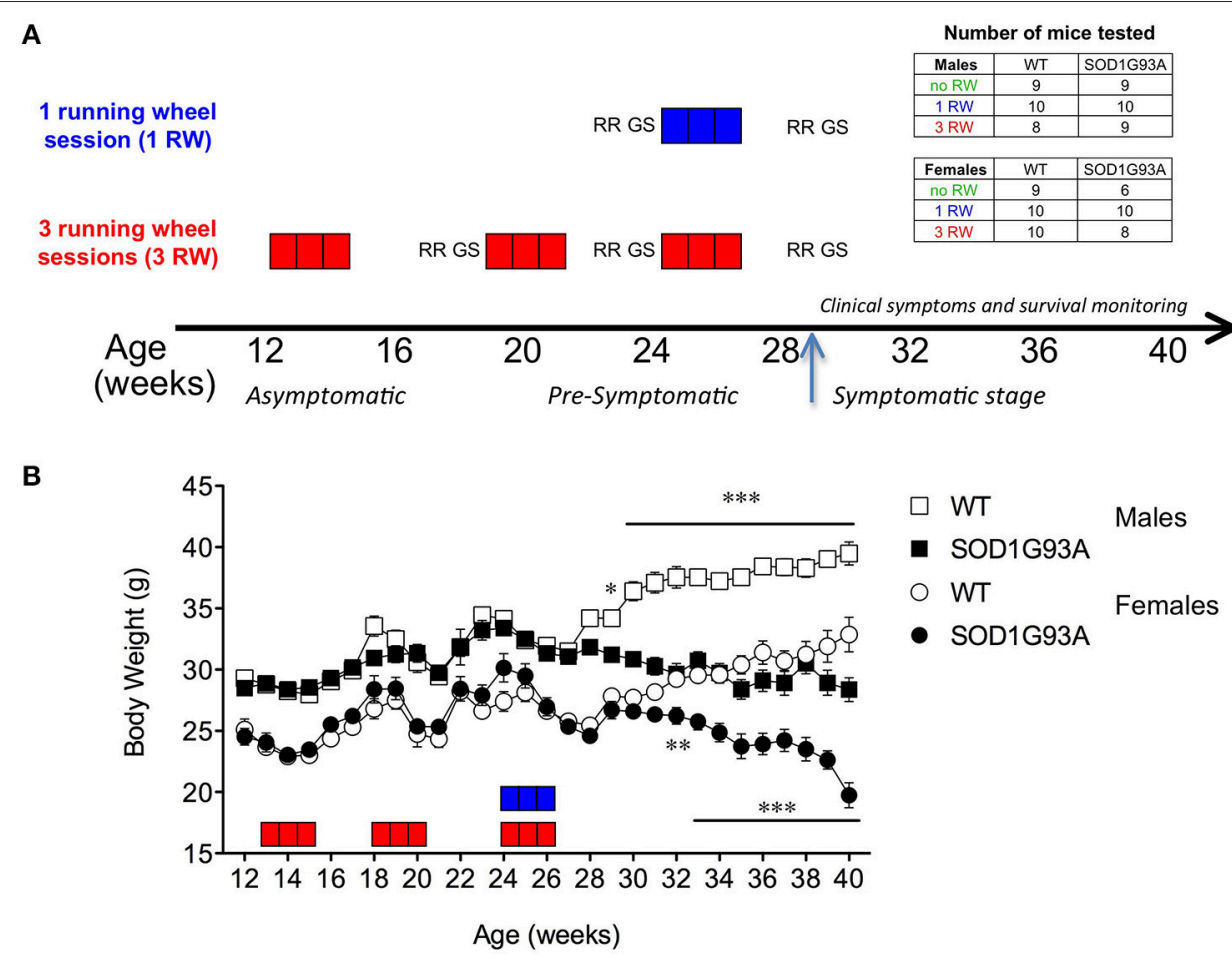

FIGURE 1 | Experimental design and body weight. (A) Experimental design: Each square box represents 1 week of running wheel (RW) exposure: in blue, group 1 RW; in red, group 3 RW. Each three squares/weeks is one session. RR, Rotarod test; GS, grip strength test. Arrow indicates appearance of disease symptoms and beginning of health and survival daily monitoring. Inset: Tables indicate the number of mice tested in each experimental group. (B) Body weight curve in grams of male and female SOD1G93A low-copy and WT littermates across experimental window (12-40 weeks of age). Number of animals varied along the study. Age 12-18 weeks (3 RW only): $n=8-10$ per sex/genotype; Age 19-30 weeks: $n=23-29$ per sex/genotype; Age 30-40 weeks: WT $n=26-29$ per sex, SOD1G93A $n=6-20$ per sex depending on survival. Asterisks indicate statistically significant differences between WT and transgenic mice in each sex group. ${ }^{\star} P<0.05$, ${ }^{\star \star} P<0.01$, ${ }^{\star \star \star} P<0.001$.

(s), where "runs" are running episodes at a velocity exceeding $30 \mathrm{rpm}(\sim 0.18 \mathrm{~m} / \mathrm{s})$; (3) average maximum speed $(\mathrm{m} / \mathrm{s})$ from daily values calculated as average of maximum speed values sampled every 5 min during the $12 \mathrm{~h}$ dark period; (4) total time on wheels (s).

\section{Rotarod}

Mice of the 3 RW group were tested on the Rotarod at 17, 23, and 29 weeks of age, while mice of the $1 \mathrm{RW}$ group were tested at 23 and 29 weeks (Figure 1A). Mice had to keep their balance on a rotating rod (3-cm diameter) set at an accelerating speed from 4 to $40 \mathrm{rpm}$ in $300 \mathrm{~s}$ (mod. 47600 apparatus; Ugo Basile, Como, Italy). To familiarize with the apparatus mice underwent a training session of 3 trials, $60 \mathrm{~s}$ each, in which the rod was kept stationary for the first trial and held at $4 \mathrm{rpm}$ for the last two trials. The next day, for 4 consecutive days, mice were tested over 3 trials/day with an inter-trial interval of $\sim 30 \mathrm{~min}$. A maximum of three mice were placed on the rod at the same time. The latency to fall from the rotating rod was recorded in each trial. If a mouse was passively rotating on the rod (i.e., clinging) the number of passive rotations were counted. For each day data were expressed as mean latency to fall minus $1 \mathrm{~s}$ "penalty" for each passive rotation (Marazziti et al., 2013). Mean 4-days latency was considered for analysis.

\section{Grip Strength}

Mice of the $3 \mathrm{RW}$ group were tested on the grip strength meter apparatus (Bioseb, France) at age 18, 24, and 30 weeks, while mice of the 1 RW group were tested at 24 and 30 weeks (Figure 1A). The mouse was held gently by the base of its tail over the top of the grid enabling forelimbs and hindlimbs to grip the grid. With its torso in a horizontal position the mouse was pulled back steadily until the grip was released down the complete length of the grid. The propensity is that the mouse will cling onto the grid until it can no longer resist the increasing force, before it is released (Mandillo et al., 2008). The grip strength meter digitally displays the maximum force applied as the peak tension (in grams) once the grasp is released. The mean of five consecutive trials was taken as an index of forelimb and hindlimb grip strength. Mice were given an inter-trial interval of about $60 \mathrm{~s}$. Body weight was taken at the end for further analyses.

\section{Body Weight, Symptoms, and Humane Endpoint Assessment}

Body weight (BW) was measured weekly from age 12 weeks (Figure 1B). From age 30 weeks, daily monitoring of disease symptoms was performed: tremors, hind limb extension reflex loss, abnormal gait and posture, bradykinesia, and paralysis were annotated. 
Extra wet food was provided as needed. Humane endpoint was set at limb paralysis onset or when weight drop exceeded $25 \%$ from peak body weight. Disease onset was defined as appearance of symptoms and the time at which the mouse permanently starts to lose weight (age at peak body weight). Mice that reached humane endpoint were sacrificed by carbon dioxide inhalation.

\section{Statistical Analysis}

A repeated-measures ANOVA (RM ANOVA) was performed on most datasets with Genotype, Sex and Exercise as betweensubject factors and Age or Time as within-subject factors. Posthoc analysis was performed where possible.

Three independent principal component analyses (PCA) (Giuliani, 2017) were performed including all animals (total $n=$ $108)$, only running mice $(n=75)$, and only mutant mice $(n=$ 52), respectively. In turn, for each of the above groups, PCA was applied separately for three groups of variables, namely: (1) Body weight, rotarod latency, grip strength; (2) wheel-running distance and run duration, time on wheels; (3) age (days) at peak BW (disease onset), survival and duration of disease (survival days minus disease onset).

Pearson's correlations and three-way ANOVAs with Genotype, Sex and Exercise as main factors were then separately performed on the extracted components. Unpaired $t$-tests and Chi-square tests were also performed to check specific hypotheses.

Subjects whose individual values differed more than 2 standard deviation units in module from the group average were considered outliers and were excluded from analysis. Significance level was set at $P<0.05$. Data are presented as mean \pm s.e.m. All statistics were run using the StatView 5.0 PowerPC, SAS System 9.1 (SAS Institute Inc., Cary, NC) and Prism 5.0a (GraphPad Software Inc., La Jolla, CA, USA) software packages.

\section{RESULTS}

\section{Exercise Induces Significant Body Weight Changes in Male SOD1G93A Low-Copy Mice}

Figure 1B shows the time course of body weight across the experimental window, from 12 to 40 weeks of age in male and female SOD1G93A low-copy mice and their WT littermates. SOD1G93A low-copy mice showed significantly lower body weight compared to WT starting from 29 weeks of age in males $(P<0.05)$ and from 32 weeks of age in females $(P<0.01)$, suggesting a delayed disease onset in female mice.

Exercise exposure affected body weight in a gender and genotype-dependent manner (Figure 2). Male SOD1G93A lowcopy mice exposed to 3 sessions of running activity showed a lower body weight compared to mice of the same genotype that had only one session of running or no running exposure (Figure 2A). This difference due to exercise exposure further increased in SOD1G93A with the progression of the disease when observed at the symptomatic stage. On the other hand, body weight of female SOD1G93A low-copy mice did not show overt decrease after prolonged exercise exposure, compared to $1 \mathrm{RW}$

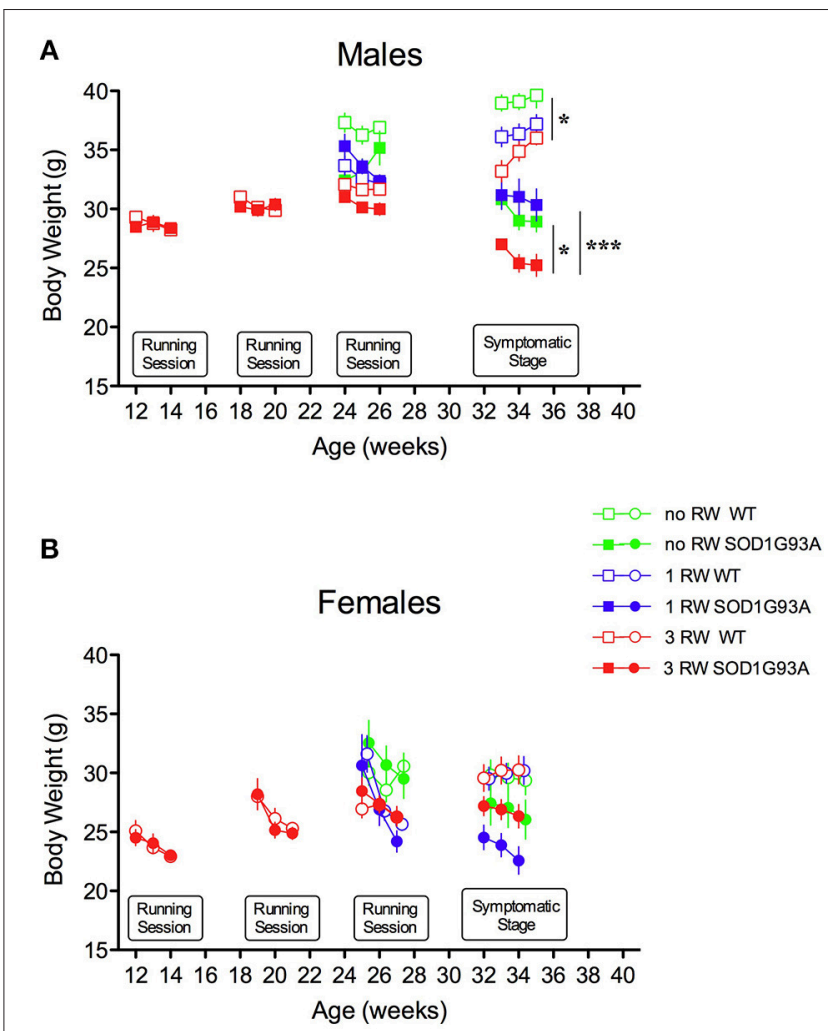

FIGURE 2 | Effect of running wheel exercise on body weight. (A) Effect of exercise on body weight of male mice during running sessions and at symptomatic stage. ANOVA, Running session at 24-26 weeks: Genotype effect, $F_{(1,47)}=4.69, P=0.035$; Exercise effect, $F_{(2,47)}=12.53, P<0.0001$, Genotype $\times$ Exercise, $F_{(2,47)}=4.853, P=0.012$. ANOVA, Symptomatic stage (age 32-34 weeks): Genotype effect $F_{(1,49)}=120.3, P<0.0001$; Exercise effect $F_{(2,49)}=10.74, P=0.0001 . n=8-10$ per group. ${ }^{\star} P<0.05$ no RW vs. 3 RW in WT and SOD1G93A low-copy mice, ${ }^{\star \star \star} P<0.0011$ RW vs. 3 RW in SOD1G93A low-copy mice, Bonferroni post-hoc test. (B) Effect of exercise on body weight of female mice during running sessions and at symptomatic stage. ANOVA, Running session at 24-26 weeks: Exercise effect, $F_{(2,43)}=4.446, P=0.017$. ANOVA, Symptomatic stage (age 32-34 weeks): Genotype effect, $F_{(1,43)}=17.18, P=0.0002 . n=6-10$ per group.

(Figure 2B). It appeared that repeated exercise sessions (3 RW group) canceled sex differences in body weight of transgenic mice (male and female mice weighted, respectively, g 26.03 $( \pm 0.46)$ and $g 26.81( \pm 0.86)$ at the symptomatic stage $)$. As expected, mice not exposed to running wheels weighed more than exercised mice. This difference was more pronounced in males.

\section{Repeated Exercise Reduces Survival of Male SOD1G93A Low-Copy Mice}

Different levels of exercise also affected survival of SOD1G93A low-copy mice in a sex-dependent fashion (Figure 3). Males exposed to higher level of exercise ( $3 \mathrm{RW}$ ) and females exposed to only one session (1 RW) had a shorter survival than animals never exposed to a running wheel (Figure 3A, Log-rank test $P=$ 0.0075; Figure 3C, Log-rank test $P=0.02$ ). 

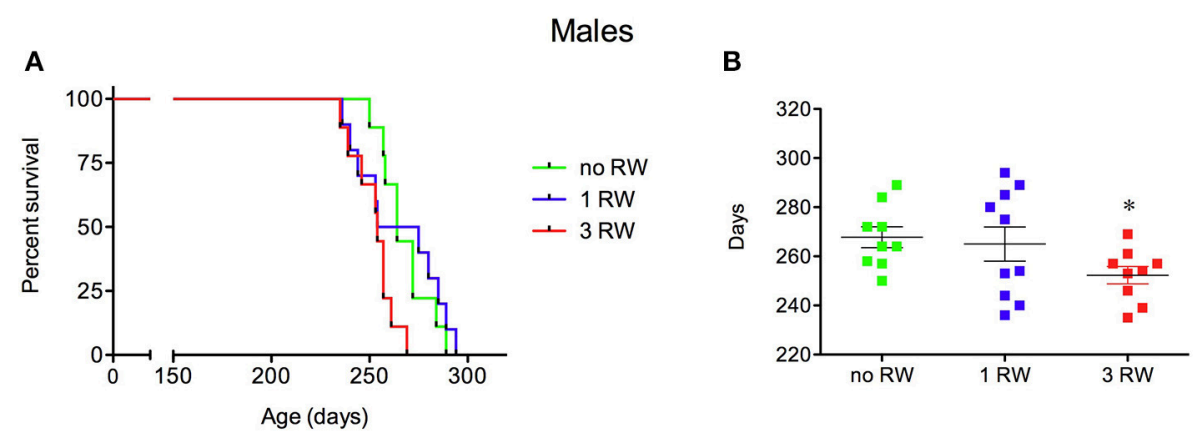

Females
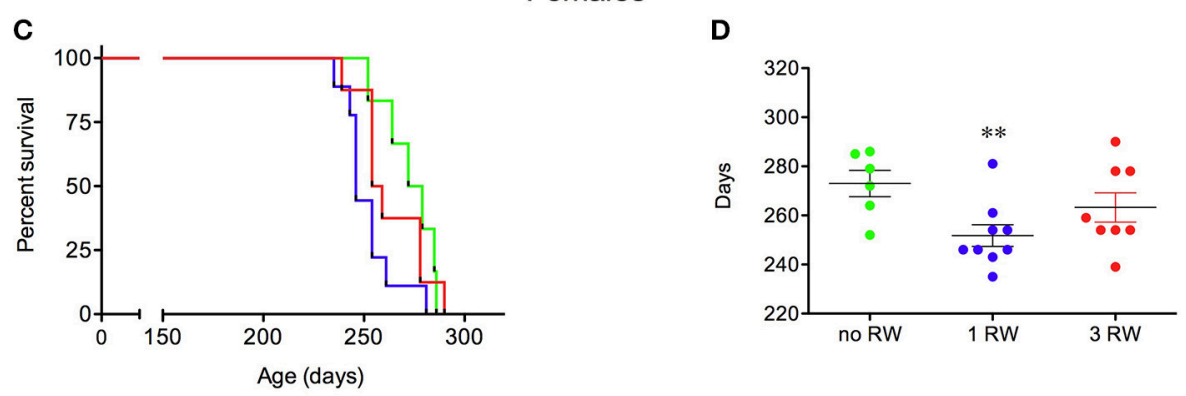

FIGURE 3 | Effect of exercise experience on survival of SOD1G93A low-copy mice. (A-C) Kaplan-Meier survival curves for male (A) and female (C) mice from the exercise groups (1 and $3 \mathrm{RW}$ ) and from a group never exposed to running wheel exercise (no RW). (B-D) Scatter plot of survival days (mean \pm s.e.m.) for male (B) and female (D) mice from the three experimental groups. ${ }^{*} P<0.05$, no RW vs. 3 RW, $t$-test; ${ }^{* \star} P<0.01$, no RW vs. 1 RW, $t$-test. $n=6-10$ per group.

In particular, male SOD1G93A low-copy mice of the $3 \mathrm{RW}$ group had a shorter life span (252.3 \pm 4 days) than the animals exposed to only one session of running activity (1 RW, $265 \pm$ 7 days) or mice that were never exposed to the running wheel (no RW, $267.8 \pm 4$ days) (Figure 3B). Females (Figure 3D) had a shorter life span when exposed to 1 RW session (251.8 \pm 4 days) compared to the no RW group ( $273 \pm 5$ days) or the 3 RW (263.3 \pm 6 days).

\section{Previous Running Experience Improves Male SOD1G93A Mice Running Performance}

Exercise experience also affected running performance in a genotype and sex-dependent manner (Figures 4, 5). Running wheel activity was compared between the $1 \mathrm{RW}$ and $3 \mathrm{RW}$ groups at the age of 24-26 weeks. Previous exercise experience (3 RW) seemed to bring running performance of transgenic mice to levels comparable to WT in both males and females. Performance on the complex wheel during the third week was reduced similarly in all groups (Figure 4). In particular, running performance (distance, run duration and speed) was analyzed during the second week of the session as in a previous study (Mandillo et al., 2014), we found that animals improved their performance throughout the first week of wheel running, whereas this reached a stable plateau and remained consistent over the second week (Figure 4, week2 and Figure 5). SOD1G93A lowcopy male mice of the $3 \mathrm{RW}$ group compared to the $1 \mathrm{RW}$ group, showed higher levels of distance traveled (Figures 4, 5A), run duration (Figure 5C) and maximum speed reached (Figure 5E). No such differences were observed in WT males (Figures 5A,C,E). Overall, female mice ran more (Figure 4) and for longer bouts than males [Figures 5B,D; ANOVA Sex factor: Distance $F_{(1,67)}=46.029, P<0.0001$; Run Duration $F_{(1,67)}=$ 4.592, $P<0.05$ ] but both sexes showed similar running speeds (Figures 5E,F).

\section{Rotarod and Grip Strength Performance Is Affected by Exercise Experience}

In the rotarod test, animals of the $3 \mathrm{RW}$ group showed longer latencies to fall compared to the $1 \mathrm{RW}$ group in both males and females at 23 weeks of age [Figures 6A,B; ANOVA Exercise factor: $\left.F_{(1,67)}=26.621, P<0.0001\right]$. At that age (23 weeks) mice of the $3 \mathrm{RW}$ and $1 \mathrm{RW}$ groups had run for 2 or none RW sessions, respectively. Conversely, no effect of exercise was observed on the rotarod at 29 weeks of age but at this time point transgenic mice performed worse than WT [Figures 6A,B; ANOVA Genotype factor, $F_{(1,67)}=4.398, P=0.03$ ]. A statistically significant interaction between Age and Exercise was in fact found in both sexes [RM ANOVA, $F_{(1,67)}=24.622, P<0.0001$ ].

Grip strength performance was positively affected by repeated exercise only in females [Figure 6D; RM ANOVA, Sex $\times$ Exercise group $\left.F_{(1,67)}=15.344, P=0.0002\right]$. As expected, transgenic SOD1G93A low-copy mice showed a significant decline in grip strength at the age of 30 weeks [Figures 6C,D; ANOVA, $\left.F_{(1,67)}=37.722, P<0.0001\right]$. 


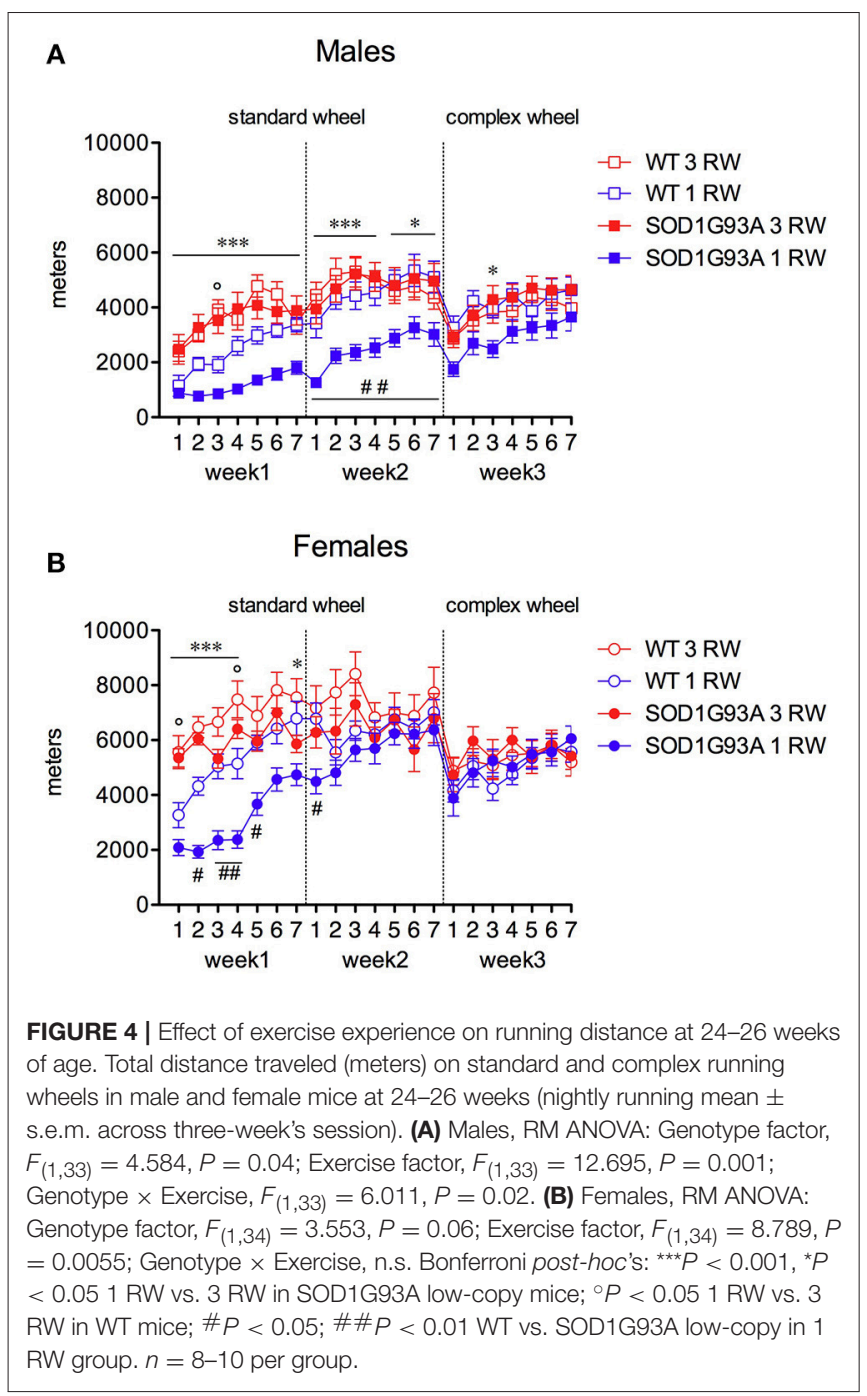

\section{Repeated Exercise Worsen Disease Prognosis in Male SOD1G93A Mice}

To have a comprehensive view of the results described so far, a Principal Component Analysis (PCA) was performed on a selection of variables from the various tests and measures. A first analysis was conducted on all mice (both running and nonrunning) by considering the following variables: body weight at age 22-24 weeks (pre-symptomatic stage) and 32-34 weeks (symptomatic stage), rotarod latency to fall at age 23 and 29 weeks, grip strength at age 24 and 30 weeks. From the PCA, three components (factors) emerged accounting for $78.2 \%$ of the total variance: on scree-test inspection (Giuliani, 2017) this three component solution can be considered a reliable global representation of the data set. Table 1 illustrates the component loadings pattern (loadings correspond to the Pearson correlation between original variables and components). The first Principal Component (PC1) accounted for $35.8 \%$ of the variance, it is a "size" component (Jolicoeur and Mosimann, 1960) with all positive loadings for the variables related to physical strength.
PC1 positively correlates with both rotarod and grip tests and can thus be interpreted as the "force" related to body mass. The second component (PC2) accounted for $29 \%$ of the variance and gives a different perspective on the effect of physical strength (indicated by body weight with high and positive loadings) on rotarod performance (with negative loadings). This inverse relation between body weight and rotarod performance can be interpreted as "motor coordination or agility" that, as expected, has no relation with grip strength performance (low loadings). In other words the same measure (body weight) exerts two opposite (and mutually independent) effects on rotarod performance: a positive one $(\mathrm{PC} 1)$ related to the increase in physical strength and a negative one (PC2) related to the decrease in agility.

The third component (PC3) accounted for $13.4 \%$ of the variance and is associated to a singularity of the grip test only at 24 weeks not easy to interpret.

When further analyzing by ANOVA the two PCs (PC1 = "force"; PC2 = "coordination"), we found statistically significant effects as for Genotype, Sex, Exercise, and Sex $\times$ Exercise for the component "force" (Supplementary Table 1). For the "coordination" component, a statistically significant effect was found only for the factors Sex and Exercise but not for Genotype.

The distribution of the animals in the PC1/PC2 (strength/coordination) plane in terms of their experimental groups is reported in Figure 7. SOD1G93A individuals are mostly distributed in the "low strength" quadrants (Figure 7A). Similarly, more females than males fall in the "low strength" quadrant (Figure 7B). As somewhat expected, individuals exposed to repeated exercise are mostly distributed in the "high strength/high coordination" quadrants, while the majority of no running mice fall in the "low strength/low coordination" quadrants (Figure 7C).

In Figure 8 are depicted the distributions of WT and SOD1G93A low-copy mice of the three groups (No RW, 1 RW, $3 \mathrm{RW}$ ) in the PC1 and PC2 principal components space and the percentage of individuals of each group that occupy each quadrant. In particular, WT mice exposed to $3 \mathrm{RW}$ exercise sessions are found mainly (83\%) on Q4 (High strength-High Coordination) quadrant, compared to only $41 \%$ of the $3 \mathrm{RW}$ mutant mice. Regardless of exercise exposure, most of the mutant mice occupy Q1 and Q3 (Low strength) quadrants while the WT are found mainly in the Q2 and Q4 (High strength) quadrants. Additionally, mutant mice of the No RW group fall mainly (80\%) in the Q1 (Low strength-Low Coordination) quadrant. Chisquare comparisons among all groups are statistically significant (Chi-square: $\mathrm{WT}=31.96, P<0.0001$; $\mathrm{SOD} 1 \mathrm{G} 93 \mathrm{~A}=26.81, P=$ 0.0002).

We then analyzed only the animals that had access to the running wheel for one (1 RW) or three sessions (3 RW). The PCA performed on the running wheel variables (distance, run duration and time on wheels) revealed only one component that accounted for $69 \%$ of the variance and it was interpreted as "running wheel performance" (with the three factor loadings all positive and $>0.77$ ). The ANOVA on this component showed statistically significant effects of Genotype, Sex, and Exercise (Supplementary Table 1). 
Males

A

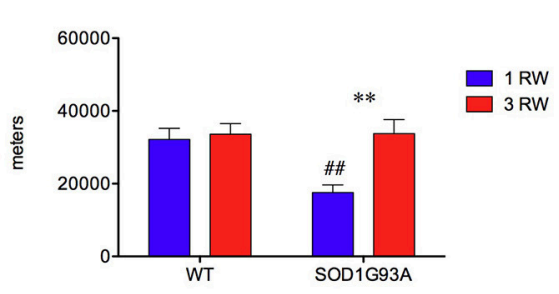

Females

B

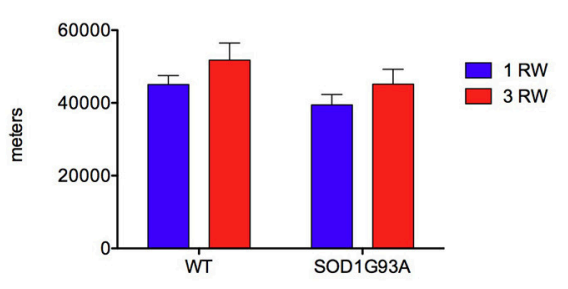

\section{Run duration}

C

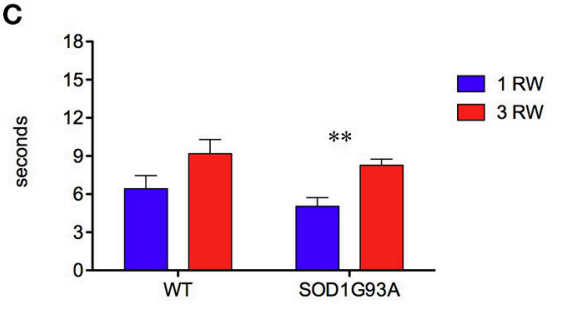

D

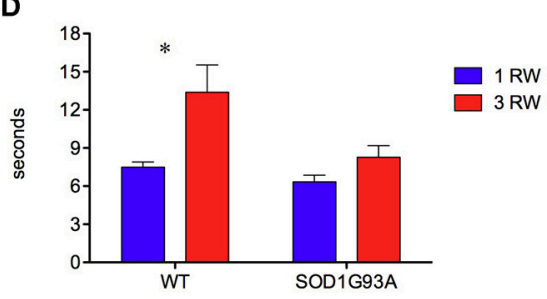

Maximum speed

E

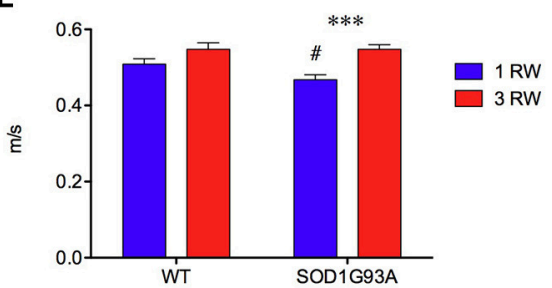

$\mathbf{F}$

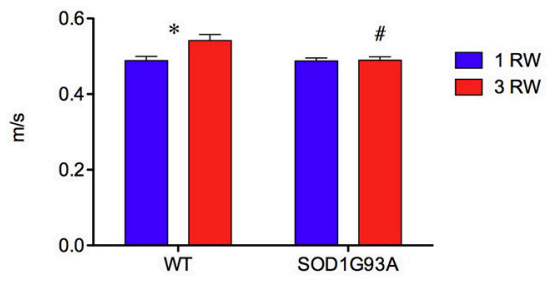

FIGURE 5 | Effect of exercise experience on running parameters during week 2 at age 24-26 weeks. (A,B) Total distance traveled (meters) on running wheels in male and female mice (mean \pm s.e.m. of session's second week). (A) Males, ANOVA: Genotype factor, $F_{(1,33)}=5.674, P=0.023$; Exercise factor, $F_{(1,33)}=8.502, P=$ 0.0063; Genotype $\times$ Exercise, $F_{(1,33)}=5.906, P=0.02$. $t$-test: ${ }^{\star \star} P=0.00141 \mathrm{RW}$ vs. 3 RW in SOD1G93A low-copy mice; \#\#P=0.001 WT vs. SOD1G93A low-copy in 1 RW group. (B) Females, ANOVA: All factors are n.s. (C,D) Mean duration (seconds) of running episodes (>30 rpm) on running wheels in male and female mice (mean \pm s.e.m. of session's second week). (C) Males, ANOVA: Exercise factor, $F_{(1,33)}=12.179, P=0.0014$, other factors are n.s. $t$-test: ${ }^{\star \star} P=0.00141$ RW vs. 3 RW in SOD1G93A low copy mice. (D) Females, ANOVA: Genotype factor, $F_{(1,34)}=6.259, P=0.017$; Exercise factor, $F(1,34)=9.774, P=0.0036$. ${ }^{\star} P=$ 0.0141 RW vs. 3 RW in WT mice. (E,F) Average maximum speed (m/sec) of running in male and female mice (mean \pm s.e.m. of session's second week). (E) Males, ANOVA: Exercise factor, $F_{(1,33)}=18.311, P=0.0002 ; t$-test: ${ }^{\star \star \star} P=0.00031 \mathrm{RW}$ vs. 3 RW in SOD1G93A low-copy mice; \# $P=0.04$ WT vs. SOD1G93A low-copy in 1 RW group. (F) Females, ANOVA: Genotype factor, $F_{(1,34)}=5.022, P=0.03$; Exercise factor, $F_{(1,34)}=5.226, P=0.028$; Genotype $\times$ Exercise, $F_{(1,34)}=4.499$, $P=0.04$. $t$-test: ${ }^{\star} P=0.0161 \mathrm{RW}$ vs. $3 \mathrm{RW}$ in WT mice; $\# P=0.02 \mathrm{WT}$ vs. SOD1G93A low-copy in $3 \mathrm{RW}$ group. $n=8-10$ per group.

Finally, we focused only on the SOD1G93A low-copy mice by considering the parameters related to the disease: survival, days at peak body weight (i.e., disease onset) and duration of disease. From the PCA, two components clearly emerged, with the first accounting for the $67 \%$ and the second for $33 \%$ of variance. The first component is all due to the duration of the disease (Table 2) while the second is completely independent from the duration but is related to onset and survival (both high and positive loadings). The first can be interpreted as "duration" of the disease or with its "prognosis" while the second is related to the "time with no disease." Oversimplifying we could name the first as "disease-related" and the second as "life time without disease."

Interestingly, the ANOVA on these 2 components revealed statistically significant effects of Exercise and Sex $\times$ Exercise interaction only for the "disease-related" component (Supplementary Table 1), no statistically significant effects were found for the second component. Correlating the component 
A

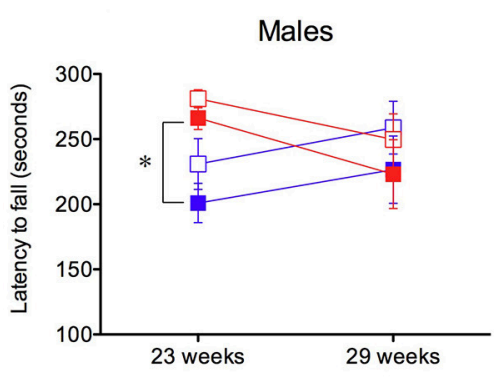

C

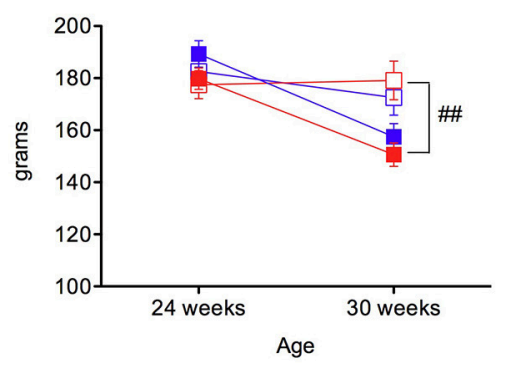

\section{Rotarod}

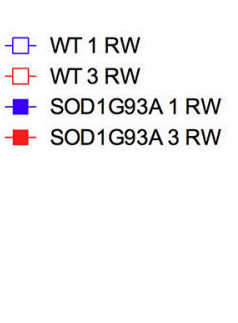

Grip test

D

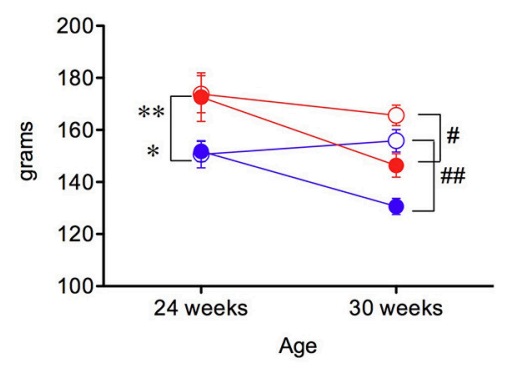

FIGURE 6 | Effect of exercise experience on Rotarod and Grip strength tests. (A,B) Rotarod test. Latency to fall (seconds, mean \pm s.e.m. of 3 trials/4 days) from rod in male and female mice at age 23 and 29 weeks. Bonferroni post-hoc tests: ${ }^{*} P<0.05$, ${ }^{*} P<0.011$ RW vs. 3 RW; $\# P<0.05$ WT vs. SOD1G93A. (C-D) Grip strength test (grams, 4 paws mean \pm s.e.m. of 5 trials) in male and female mice at age 24 and 30 weeks. Bonferroni post-hoc tests: ${ }^{*} P<0.051$ RW vs. 3 RW in SOD1G93A, ${ }^{\star \star} P<0.011$ RW vs. 3 RW in WT; \# $P<0.05$ WT vs. SOD1G93A in 3 RW, \#\# $P<0.01$ WT vs. SOD1G93A in 3 RW males and in 1 RW females.

TABLE 1 | Principal component analysis on all mice data.

\begin{tabular}{llll}
\hline & \multicolumn{3}{c}{ Components (variance explained) } \\
\cline { 2 - 4 } Variables & PC1 (35.8\%) & PC2 (29\%) & PC3 (13.4\%) \\
\hline $\begin{array}{l}\text { Body Weight 22-24 } \\
\text { weeks }\end{array}$ & 0.400 & $\mathbf{0 . 7 9 6}$ & 0.012 \\
$\begin{array}{l}\text { Body Weight 32-34 } \\
\text { weeks }\end{array}$ & $\mathbf{0 . 6 8 4}$ & 0.494 & -0.354 \\
$\begin{array}{l}\text { Rotarod latency 23 } \\
\text { weeks }\end{array}$ & 0.543 & $-\mathbf{0 . 6 7 5}$ & 0.140 \\
$\begin{array}{l}\text { Rotarod latency 29 } \\
\text { weeks }\end{array}$ & $\mathbf{0 . 6 0 5}$ & $-\mathbf{0 . 5 8 2}$ & -0.114 \\
Grip strength 24 weeks & $\mathbf{0 . 5 8 1}$ & 0.221 & $\mathbf{0 . 7 5 3}$ \\
Grip strength 30 weeks & $\mathbf{0 . 7 2 2}$ & -0.092 & -0.287
\end{tabular}

Body weight, rotarod and grip test variables from all mice tested $(n=108)$. A three components solution was obtained, accounting for $78.2 \%$ of total variance. PC1 was interpreted as "force" related to body mass, PC2 as "coordination or agility" unrelated to strength and PC3 as a singularity of the grip strength at age 24 weeks. Data are factor loadings (in bold $\geq|0.6|$ ).

of "running wheel performance" with the "disease-related" component in the repeated exercise group (Figure 9) we found that in mutant males, the higher the RW performance the poorer the prognosis (Males: $r^{2}=0.68, P=0.0065$; Females: $r^{2}=0.38$, $P=0.1)$ and that disease prognosis was worse in mutant males than females.

\section{DISCUSSION}

In our study we found that prolonged exercise exposure had a negative impact predominantly in male SOD1G93A low-copy mice. Namely, repeated wheel running reduced survival, exacerbated body weight decline and aggravated disease prognosis.

Even if exercise had deleterious effects on body weight and survival of transgenic male mice, previous running experience had, as expected, a positive effect on both running (Figure 4A) and rotarod performance (Figure 6A) in a similar way in the two genotypes, possibly indicating a training-induced phenomenon. In fact, beside the well-known positive influence of exercise on cognition reported in both animal and human studies (Hötting and Röder, 2013), also rotarod performance has been found improved in rats after prolonged voluntary wheel running due to better motor skill learning (Buitrago et al., 2004). In transgenic female mice repeated exercise induced a poor performance in the rotarod at 29 weeks while grip strength seemed affected positively by previous exercise experience at 24 weeks only in females (Figures 6C,D). Indeed we observed that the effects of voluntary exercise vary widely depending on the type of motor function or pathological stage. In fact, wheel running, rotarod and grip strength tests, measure different aspects of motor function (Mandillo et al., 2014) and they are not necessarily affected in the same way in the two sexes and in their interaction with the disease. The use of PCA allowed us to appreciate and 
A

Genotype
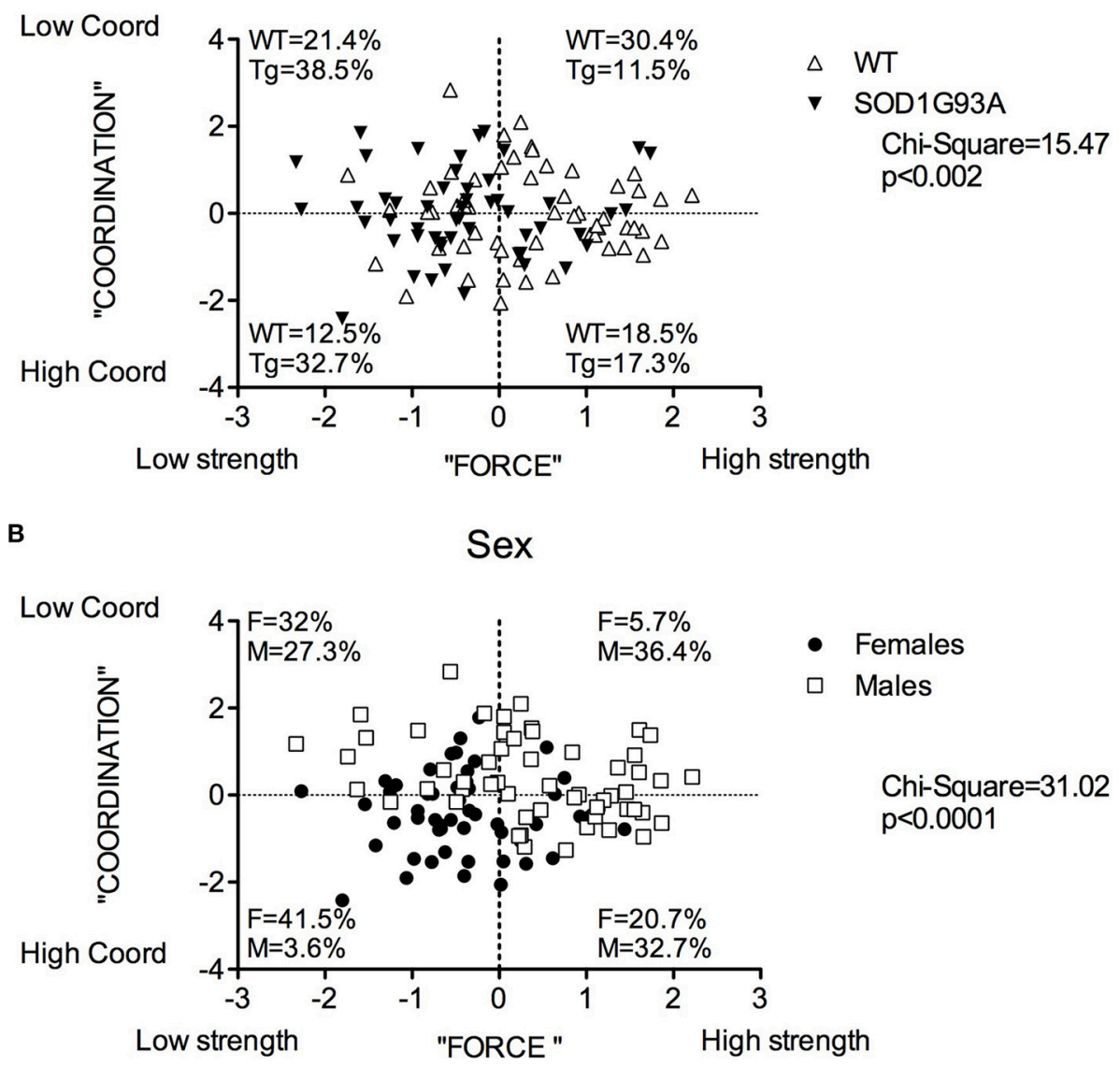

C

Exercise group

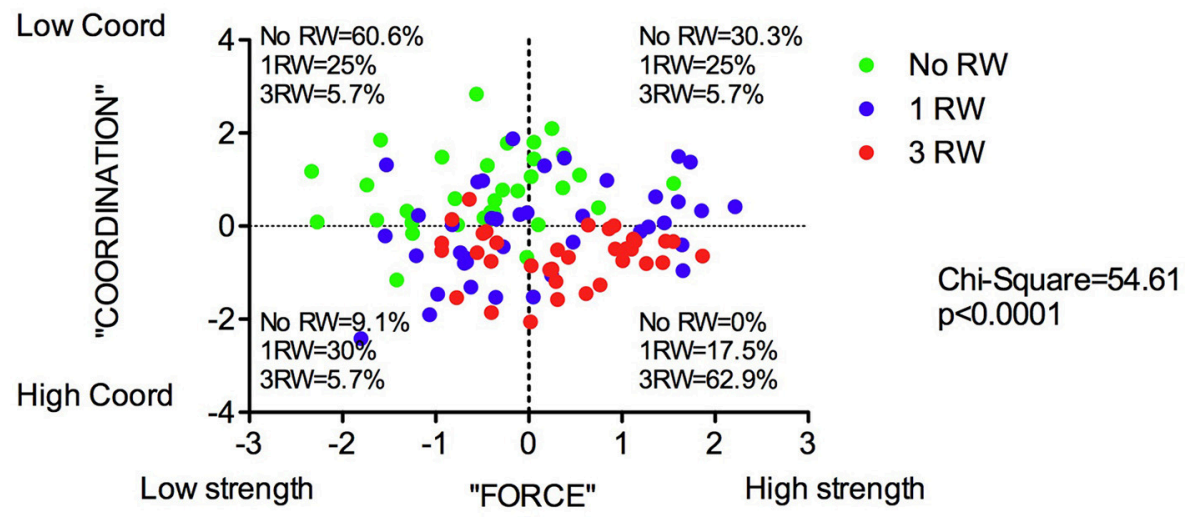

FIGURE 7 | Distribution of mice in the PC1 "Force" and PC2 "Coordination" principal components space for each between subjects factor: Genotype, Sex and Group. Distribution and percentage of individuals of $\mathbf{( A )}$ the two genotypes, (B) the two sexes, (C) the three exercise groups in the four quadrants subdividing the "Force"/"Coordination" space. Chi-square values are significant for each factor.

interpret the impact of exercise exposure on different outcomes as body weight, motor performance, and disease progression to finally discriminate their relative contribution in a pathological condition as ALS in the two sexes. Most importantly it takes in to account the large individual variability observed in this mouse model. Also in humans, different types of exercise may 
A

WT

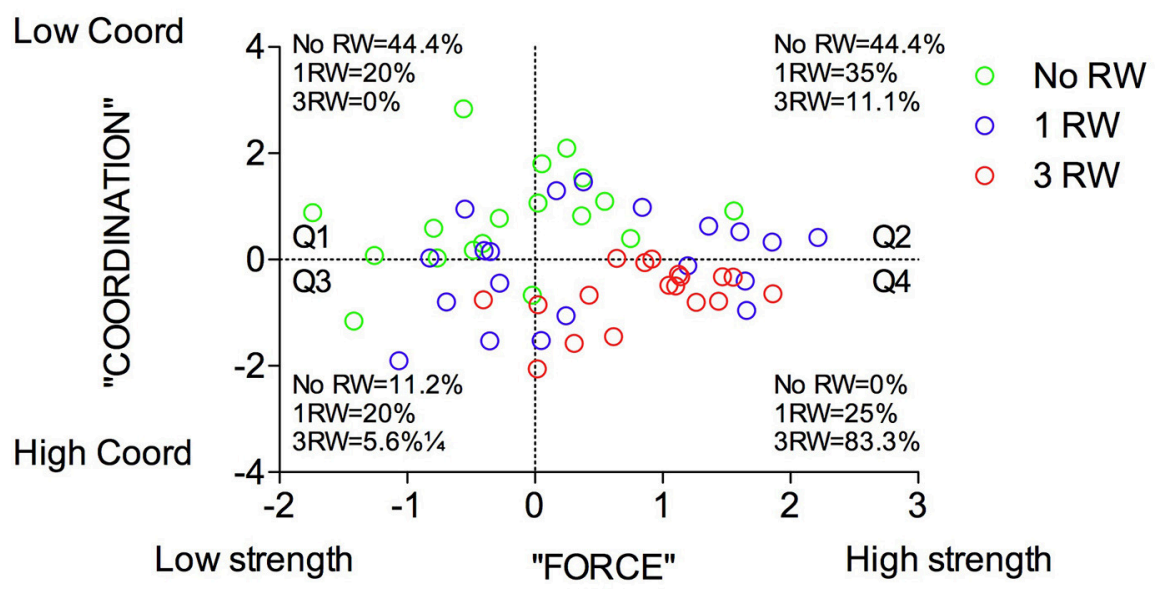

B

SOD1G93A

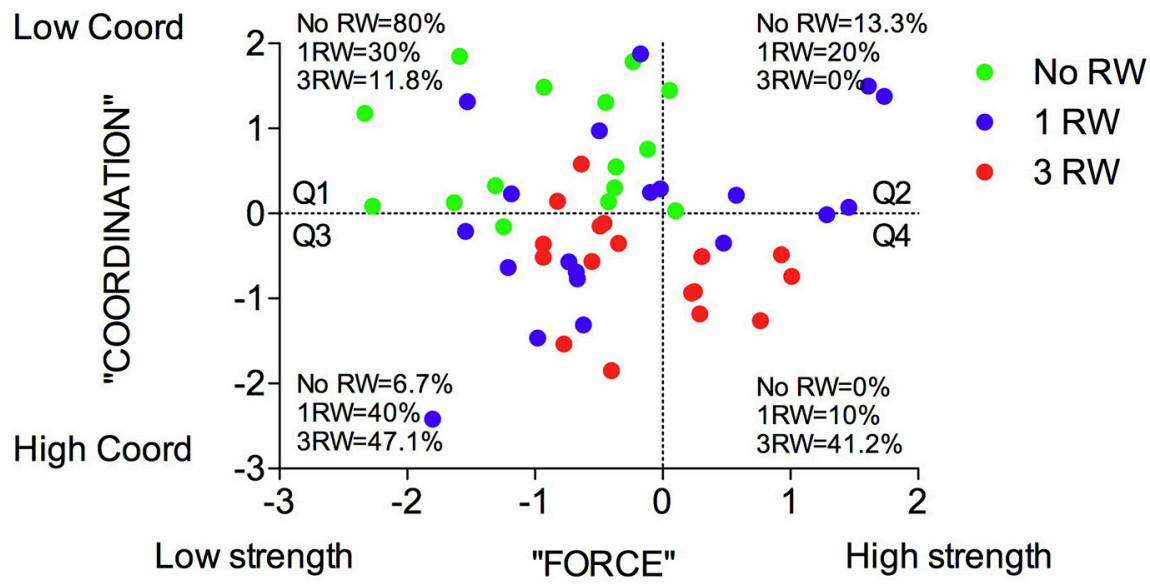

FIGURE 8 | Distribution of mice from the three exercise groups in the PC1 "Force" and PC2 "Coordination" principal components space. (A) Wild-type and (B) SOD1G93A mice distribution and percentage of individuals of the three exercise groups in the four quadrants subdividing the "Force"/"Coordination" space.

have different impact on the health of individuals depending on their sex, genetic predisposition, life style, and past experiences (Hawley et al., 2014; Rosenfeld, 2017).

Some epidemiological evidences indicate a higher risk to develop ALS associated to intense exercise or professional sports, e.g., soccer, American football, baseball (Veldink et al., 2005; Beghi et al., 2010; Beghi, 2013). However, reports on the effects of exercise in ALS patients are rather controversial (Lisle and Tennison, 2015; Lacorte et al., 2016). Part of this dispute is due to non-standardized exercise protocols and paucity of largescale human studies. In animal studies investigating the effects of exercise on ALS, very different protocols are used in terms of exercise type and duration. Most of them employ forced exercise protocols (i.e., treadmill and forced swim) that also induce a substantial amount of stress in the animal and are not so suitable to mimic human activities. We propose that voluntary exercise as the home cage wheel running used in our study, is a better model of human leisure physical activity.

While some studies found beneficial or no effect of moderate exercise on running wheels (Liebetanz et al., 2004; Kaspar et al., 2005; Bennett et al., 2014) only a few reported detrimental effects of intense forced exercise using the treadmill (Mahoney et al., 2004; Carreras et al., 2010).

Our study is the first demonstrating deleterious effects of voluntary prolonged running wheel activity. Moreover, we demonstrated that higher running wheel performance significantly correlates with poor prognosis only in mutant males. As shown in the PCA, exercise is somewhat beneficial in 
TABLE 2 | Principal component analysis on SOD1G93A mice disease-related data.

\begin{tabular}{lll}
\hline & \multicolumn{2}{c}{ Components (variance explained) } \\
\cline { 2 - 3 } Variables & PC1 (67\%) & PC2 (33\%) \\
\hline $\begin{array}{l}\text { Age at peak BW } \\
\text { (onset) }\end{array}$ & $-\mathbf{0 . 6 1 7}$ & $\mathbf{0 . 7 8 7}$ \\
$\begin{array}{l}\text { Disease Duration } \\
\text { Survival }\end{array}$ & $\mathbf{0 . 9 9 9}$ & 0.003 \\
& $\mathbf{0 . 7 9 5}$ & $\mathbf{0 . 6 0 6}$
\end{tabular}

Days at peak body weight (BW), i.e. age at disease onset, disease duration and survival variables from SOD1G93A low-copy mutant mice $(n=52)$. A clearly bipartite two components solution was obtained, accounting for $100 \%$ of total variance. PC1 was interpreted as "disease duration/prognosis," PC2 as "life time without disease" unrelated to duration. Data are factor loadings (in bold $>|0.6|$ ).

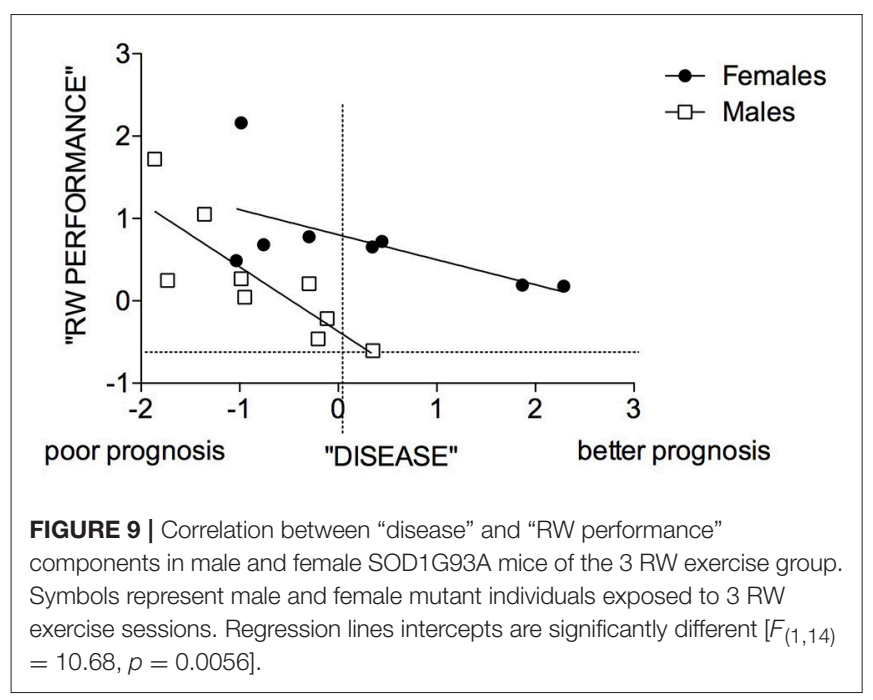

general but can be detrimental if associated to a disease related mutation. In humans, a genetic predisposition to physical fitness could be associated with higher risk of ALS (Mattsson et al., 2012; Huisman et al., 2013). Although proof of the association between physical activity and risk of ALS is still under debate (Lacorte et al., 2016), a recent survey demonstrated a positive association between sporadic ALS and long-term physical activity participation (Harwood et al., 2016).

We also showed clear sex differences in the impact of voluntary exercise on disease outcome with SOD1G93A females being less affected by prolonged physical activity but showing decreased survival after moderate exercise (1 RW) (Figures 3C,D). This could be due to their exposure to exercise for the first time at an age (24-26 weeks) that corresponds in this group to a critical phase of symptom onset, i.e., associated with a higher body weight drop (-20.9\%) compared to $3 \mathrm{RW}(-7.5 \%)$ and to males ( $1 \mathrm{RW}:-8.3 \%$; $3 \mathrm{RW}:-3.5 \%$ ). Nevertheless, females showed higher running activity than males but this did not seem to correlate with disease prognosis (Figure 9). A few other studies evaluated the effect of exercise on SOD1G93A male and female mice. They found that moderate treadmill forced exercise was beneficial only in males (Kirkinezos et al., 2003), only in females
(Veldink et al., 2003) or, similarly to our findings, high intensity exercise was detrimental only in males (Mahoney et al., 2004). Moreover, we showed that at symptomatic stage mutant males lost more body weight than females after prolonged exercise, possibly due to sex differences in muscle metabolism. In fact, an additional explanation for the negative impact of this type of exercise in ALS could be due to unbalanced energy metabolism (Dupuis et al., 2011; Ferri and Coccurello, 2017; Vandoorne et al., 2018).

Controversial findings in both human and mouse studies could be in part attributed to poor quality data or at least excessive generalization on what is defined as exercise (Nikolaidis et al., 2012; Lisle and Tennison, 2015). Different types of exercise activities should also be considered in terms of their muscledamaging impact. For example, repeated sprints and prolonged running as opposed to cycling and horizontal running are more prone to induce muscle damage via extended alteration of redox homeostasis (Nikolaidis et al., 2012). Voluntary wheel running in mice is a continuous repetition of running bouts. The parameter Running Performance used in the PCA of this study that is inversely correlating with disease prognosis, is the combination of running distance, running bout duration and time on wheel, thus reproducing the characteristics of human repeated sprints and prolonged running exercise. On the other hand, running on a complex wheel: 1) it requires different motor skills that are not related to general locomotion and 2) it implies shorter running bouts and reduced performance (Mandillo et al., 2014). In the present study, the use of the complex wheel had minimal impact on the relation between prolonged exercise and disease prognosis.

To contribute to the understanding of the mechanisms underlying the detrimental effects of voluntary prolonged and repeated exercise on several disease aspects (onset, progression, etiology, peripheral degeneration, neuromuscular pathology, inflammation and metabolism) further studies are in progress using SOD1G93A low-copy mice with the ambitious aim of proposing possible therapeutic interventions combining life style (exercise and diet) and new drug treatments to alleviate ALS symptoms. In conclusion our findings support the current guidelines on exercise interventions in ALS in which it is recommended the use of moderate rather than vigorous exercise activities (Majmudar et al., 2014). In addition, a personalized approach should be taken in ALS treatment according to genetic profile, disease stage and gender.

\section{AUTHOR CONTRIBUTIONS}

LG and SM conceived and designed the experiments. LG performed the experiments. LG, EG, AG, and SM analyzed the data. EG and SM wrote the paper.

\section{FUNDING}

This study was supported by: European Commission FP7 Programme, Project 223263, PhenoScale (LG fellowship), CNR-Progetto di interesse strategico Invecchiamento, 
IBCN/MIUR Attività Internazionale afferente all'area di Monterotondo.

\section{ACKNOWLEDGMENTS}

We thank S. Gozzi for excellent technical support. We thank TSE Systems (Bad Homburg, Germany) for supplying the running wheel equipment (FP7 Project Phenoscale). We are grateful to

\section{REFERENCES}

Acevedo-Arozena, A., Kalmar, B., Essa, S., Ricketts, T., Joyce, P., Kent, R., et al. (2011). A comprehensive assessment of the SOD1G93A low-copy transgenic mouse, which models human amyotrophic lateral sclerosis. Dis. Model. Mech. 4, 686-700. doi: 10.1242/dmm.007237

Ajroud-Driss, S., and Siddique, T. (2015). Sporadic and hereditary amyotrophic lateral sclerosis (ALS). Biochim. Biophys. Acta 1852, 679-684. doi: 10.1016/j.bbadis.2014.08.010

Al-Chalabi, A., and Hardiman, O. (2013). The epidemiology of ALS: a conspiracy of genes, environment and time. Nat. Rev. Neurol. 9, 617-628. doi: 10.1038/nrneurol.2013.203

Al-Chalabi, A., van den Berg, L. H., and Veldink, J. (2017). Gene discovery in amyotrophic lateral sclerosis: implications for clinical management. Nat. Rev. Neurol. 13, 96-104. doi: 10.1038/nrneurol.2016.182

Alexander, G. M., Erwin, K. L., Byers, N., Deitch, J. S., Augelli, B. J., Blankenhorn, E. P., et al. (2004). Effect of transgene copy number on survival in the G93A SOD1 transgenic mouse model of ALS. Brain Res. Mol. Brain Res. 130, 7-15. doi: 10.1016/j.molbrainres.2004.07.002

Beghi, E. (2013). Are professional soccer players at higher risk for ALS? Amyotroph. Lateral Scler. Frontotemporal Degener. 14, 501-506. doi: 10.3109/21678421.2013.809764

Beghi, E., Logroscino, G., Chiò, A., Hardiman, O., Millul, A., Mitchell, D., et al. (2010). Amyotrophic lateral sclerosis, physical exercise, trauma and sports: results of a population-based pilot case-control study. Amyotroph. Lateral Scler. 11, 289-292. doi: 10.3109/17482960903384283

Bennett, E. J., Mead, R. J., Azzouz, M., Shaw, P. J., and Grierson, A. J. (2014). Early detection of motor dysfunction in the SOD1G93A mouse model of Amyotrophic Lateral Sclerosis (ALS) using home cage running wheels. PLoS ONE 9:e107918. doi: 10.1371/journal.pone.0107918

Bozzoni, V., Pansarasa, O., Diamanti, L., Nosari, G., Cereda, C., and Ceroni, M. (2016). Amyotrophic lateral sclerosis and environmental factors. Funct. Neurol. 31, 7-19. doi: 10.11138/FNeur/2016.31.1.007

Buitrago, M. M., Schulz, J. B., Dichgans, J., and Luft, A. R. (2004). Short and long-term motor skill learning in an accelerated rotarod training paradigm. Neurobiol. Learn. Mem. 81, 211-216. doi: 10.1016/j.nlm.2004.01.001

Carreras, I., Yuruker, S., Aytan, N., Hossain, L., Choi, J. K., Jenkins, B. G., et al. (2010). Moderate exercise delays the motor performance decline in a transgenic model of ALS. Brain Res. 1313, 192-201. doi: 10.1016/j.brainres.2009.11.051

Clayton, J. A., and Collins, F. S. (2014). Policy: NIH to balance sex in cell and animal studies. Nature 509, 282-283. doi: 10.1038/509282a

Dupuis, L., Pradat, P. F., Ludolph, A. C., and Loeffler, J. P. (2011). Energy metabolism in amyotrophic lateral sclerosis. Lancet Neurol. 10, 75-82. doi: 10.1016/S1474-4422(10)70224-6

Ferri, A., and Coccurello, R. (2017). What is "Hyper" in the ALS Hypermetabolism? Mediators Inflamm. 2017:7821672. doi: $10.1155 / 2017 / 7821672$

Giuliani, A. (2017). The application of principal component analysis to drug discovery and biomedical data. Drug Discov. Today 22, 1069-1076. doi: 10.1016/j.drudis.2017.01.005

Gurney, M. E., Pu, H., Chiu, A. Y., Dal Canto, M. C., Polchow, C. Y., Alexander, D. D., et al. (1994). Motor neuron degeneration in mice that express a human $\mathrm{Cu}, \mathrm{Zn}$ superoxide dismutase mutation. Science 264, 1772-1775. doi: $10.1126 /$ science. 8209258 the International Mouse Phenotyping Consortium (IMPC) for fruitful sharing of procedures and discussions.

\section{SUPPLEMENTARY MATERIAL}

The Supplementary Material for this article can be found online at: https://www.frontiersin.org/articles/10.3389/fnbeh. 2018.00275/full\#supplementary-material

Harwood, C. A. D., Westgate, K. M., Gunstone, S. M., Brage, S. D., Wareham, N. J. P., McDermott, C. J. D., et al. (2016). Long-term physical activity: an exogenous risk factor for sporadic amyotrophic lateral sclerosis? Amyotroph. Lateral Scler. Frontotemporal Degener. 17, 377-384. doi: 10.3109/21678421.2016.1154575

Hawley, J. A., Hargreaves, M., Joyner, M. J., and Zierath, J. R. (2014). Integrative biology of exercise. Cell 159, 738-749. doi: 10.1016/j.cell.2014.10.029

Hötting, K., and Röder, B. (2013). Beneficial effects of physical exercise on neuroplasticity and cognition. Neurosci. Biobehav. Rev. 37(9 Pt B), 2243-2257. doi: 10.1016/j.neubiorev.2013.04.005

Huisman, M. H., Seelen, M., de Jong, S. W., Dorresteijn, K. R., van Doormaal, P. T., van der Kooi, A. J., et al. (2013). Lifetime physical activity and the risk of amyotrophic lateral sclerosis. J. Neurol. Neurosurg. Psychiatr. 84, 976-981. doi: 10.1136/jnnp-2012-304724

Jolicoeur, P., and Mosimann, J. E. (1960). Size and shape variation in the painted turtle. a principal component analysis. Growth 24, 339-354.

Kaspar, B. K., Frost, L. M., Christian, L., Umapathi, P., and Gage, F. H. (2005). Synergy of insulin-like growth factor-1 and exercise in amyotrophic lateral sclerosis. Ann. Neurol. 57, 649-655. doi: 10.1002/ana.20451

Kirkinezos, I. G., Hernandez, D., Bradley, W. G., and Moraes, C. T. (2003). Regular exercise is beneficial to a mouse model of amyotrophic lateral sclerosis. Ann. Neurol. 53, 804-807. doi: 10.1002/ana.10597

Lacorte, E., Ferrigno, L., Leoncini, E., Corbo, M., Boccia, S., and Vanacore, N. (2016). Physical activity, and physical activity related to sports, leisure and occupational activity as risk factors for ALS: a systematic review. Neurosci. Biobehav. Rev. 66, 61-79. doi: 10.1016/j.neubiorev.2016.04.007

Liebetanz, D., Hagemann, K., von Lewinski, F., Kahler, E., and Paulus, W. (2004). Extensive exercise is not harmful in amyotrophic lateral sclerosis. Eur. J. Neurosci. 20, 3115-3120. doi: 10.1111/j.1460-9568.2004.03769.x

Liebetanz, D., and Merkler, D. (2006). Effects of commissural deand remyelination on motor skill behaviour in the cuprizone mouse model of multiple sclerosis. Exp. Neurol. 202, 217-224. doi: 10.1016/j.expneurol.2006.05.032

Lisle, S., and Tennison, M. (2015). Amyotrophic lateral sclerosis: the role of exercise. Curr. Sports Med. Rep. 14, 45-46. doi: 10.1249/JSR.0000000000000122

Mahoney, D. J., Rodriguez, C., Devries, M., Yasuda, N., and Tarnopolsky, M. A. (2004). Effects of high-intensity endurance exercise training in the G93A mouse model of amyotrophic lateral sclerosis. Muscle Nerve 29, 656-662. doi: 10.1002/mus.20004

Majmudar, S., Wu, J., and Paganoni, S. (2014). Rehabilitation in amyotrophic lateral sclerosis: why it matters. Muscle Nerve 50, 4-13. doi: 10.1002/mus.24202

Mandillo, S., Heise, I., Garbugino, L., Tocchini-Valentini, G. P., Giuliani, A., Wells, S., et al. (2014). Early motor deficits in mouse disease models are reliably uncovered using an automated home-cage wheel-running system: a crosslaboratory validation. Dis. Model. Mech. 7, 397-407. doi: 10.1242/dmm.013946

Mandillo, S., Tucci, V., Hölter, S. M., Meziane, H., Banchaabouchi, M. A., Kallnik, M., et al. (2008). Reliability, robustness, and reproducibility in mouse behavioral phenotyping: a cross-laboratory study. Physiol. Genomics 34, 243-255. doi: 10.1152/physiolgenomics.90207.2008

Marazziti, D., Di Pietro, C., Golini, E., Mandillo, S., La Sala, G., Matteoni, R., et al. (2013). Precocious cerebellum development and improved motor functions in mice lacking the astrocyte cilium-, patched 1-associated Gpr3711 receptor. Proc. Natl. Acad. Sci. U.S.A. 110, 16486-16491. doi: 10.1073/pnas.1314819110

Mattsson, P., Lonnstedt, I., Nygren, I., and Askmark, H. (2012). Physical fitness, but not muscle strength, is a risk factor for death in amyotrophic 
lateral sclerosis at an early age. J. Neurol. Neurosurg. Psychiatr. 83, 390-394. doi: 10.1136/jnnp.2010.218982

Nikolaidis, M. G., Kyparos, A., Spanou, C., Paschalis, V., Theodorou, A. A., and Vrabas, I. S. (2012). Redox biology of exercise: an integrative and comparative consideration of some overlooked issues. J. Exp. Biol. 215(Pt 10), 1615-1625. doi: $10.1242 /$ jeb.067470

Oskarsson, B., Horton, D. K., and Mitsumoto, H. (2015). Potential environmental factors in amyotrophic lateral sclerosis. Neurol. Clin. 33, 877-888. doi: 10.1016/j.ncl.2015.07.009

Rosenfeld, C. S. (2017). Sex-dependent differences in voluntary physical activity. J. Neurosci. Res. 95, 279-290. doi: 10.1002/jnr.23896

Vandoorne, T., De Bock, K., and Van Den Bosch, L. (2018). Energy metabolism in ALS: an underappreciated opportunity? Acta Neuropathol. 135, 489-509. doi: 10.1007/s00401-018-1835-X

Veldink, J. H., Bär, P. R., Joosten, E. A., Otten, M., Wokke, J. H., and van den Berg, L. H. (2003). Sexual differences in onset of disease and response to exercise in a transgenic model of ALS. Neuromuscul. Disord. 13, 737-743. doi: 10.1016/S0960-8966(03)00104-4
Veldink, J. H., Kalmijn, S., Groeneveld, G. J., Titulaer, M. J., Wokke, J. H., and van den Berg, L. H. (2005). Physical activity and the association with sporadic ALS Neurology 64, 241-245. doi: 10.1212/01.WNL.0000149513.82332.5C

Conflict of Interest Statement: The authors declare that the research was conducted in the absence of any commercial or financial relationships that could be construed as a potential conflict of interest.

The handling editor declared a shared affiliation, though no other collaboration, with one of the authors AG at the time of the review.

Copyright (c) 2018 Garbugino, Golini, Giuliani and Mandillo. This is an open-access article distributed under the terms of the Creative Commons Attribution License (CC $B Y)$. The use, distribution or reproduction in other forums is permitted, provided the original author(s) and the copyright owner(s) are credited and that the original publication in this journal is cited, in accordance with accepted academic practice. No use, distribution or reproduction is permitted which does not comply with these terms. 